\title{
Madridge
}

madridge Journal of Nanotechnology \& Nanoscience

Interconnecting Scientific World

Opinion Article

Open Access

\section{Carbon nanotubes - synthesis developmental engineering demands will overcome the health challenge of nanotoxicity and its acute mortality for humans}

\author{
Loai Aljerf ${ }^{{ }^{\star}}$ and Bayan AlHamwi2 \\ IDepartment of Basic Sciences, Faculty of Dental Medicine, Damascus University, Damascus, Syria \\ 2Department of Chemistry, Faculty of Dentistry, Syrian Private University, Damascus, Syria
}

\section{Article Info}

\author{
*Corresponding author: \\ Loai Aljerf \\ Department of Basic Sciences \\ Faculty of Dental Medicine \\ Damascus University \\ Damascus, Syria \\ E-mail: envirochrom@hotmail.com
}

Received: October 31, 2018

Accepted: November 21, 2018

Published: November 27, 2018

Citation: Aljerf L, AlHamwi B. Carbon nanotubes - synthesis developmental engineering demands will overcome the health challenge of nanotoxicity and its acute mortality for humans. Madridge $J$ Nanotechnol Nanosci. 2018; 3(2): 116-118. doi: 10.18689/mjnn-1000122

Copyright: (c) 2018 The Author(s). This work is licensed under a Creative Commons Attribution 4.0 International License, which permits unrestricted use, distribution, and reproduction in any medium, provided the original work is properly cited.

Published by Madridge Publishers

\begin{abstract}
Nanomanufacturing is the essential bridge between the discoveries of the nanosciences and real-world nanotechnology-enabled products. It is the utilization of value-added processes to control matter at the nanoscale in one, two, and three dimensions for reproducible, commercial-scale production. It encompasses bottom-up directed assembly, top-down high resolution processing, molecular systems engineering, and hierarchical integration with macro-scale systems. Advancing nanotechnology starting from the laboratory into high-volume production ultimately requires careful study of manufacturing system issues including product design, reliability and quality, process design and control, shop floor operations and supply chain management. However, nanomanufacturing processes are now key enablers to solve critical issues in the evolution of many products, fueling the innovation cycle to realize completely new products. These processes include bottom-up directed assembly, top-down high-resolution patterning and manipulation, molecular and biological systems engineering, and hierarchical integration across multiple length scales. As mentioned in one of our previous published articles, carbon nanotubes (CNTs) can be used as nanomaterial (NM) in both industrial and medical applications and they have significant promising biomedical applications, such as drug design, drug delivery, tumor therapy, tissue engineering, DNA recognition, and biosensor design. These issues bring a new feature to the discussion about purpose and mechanisms underlying the reaction of an organism with nanoparticles. The current discussion thus gives a new focus to the important aspect of nanotechnology, which can help researchers in determining the application aspects of this technology in future: the approach of biological systems to nanosized particles.
\end{abstract}

Keywords: Carbon Nanotubes; Supply Chain Management; Biomedical Applications; Ultrafine Particles; Health Effects; Toxic Response; Risk Management Systems

\section{Carbon nanotubes engineering under our scope}

In industry year, the field of nanomanufacturing engineering which aims to contribute to a wider understanding of how our economy and standard of living critically depend on those who design, manufacture and sell the nanoproducts of high quality necessary to compete in world markets. The two main thrusts worldwide, in nanomanufacturing engineering are Automation-in particular, computer integrated, 
flexible nanomanufacture to reduce overall cost and lead time and in which flexible manufacturing systems (FMS) and computer integrated manufacture (CIM) are crucially important technologies manufacture with higher precisionon which a wide range of advanced technology products are totally dependent-and in which precision engineering, microengineering, and nanotechnology are generally less well understood and practiced than by our main international competitors.

However, the potential health risks associated with nanotechnology have received considerable attention over recent years. Largely based on research into the toxicity of 'ultrafine particles', concerns have been expressed that engineered nanosized and nanostructured particles released into the workplace will lead to health risks not predicted within current paradigms. Such is the interest in the potentially unique behavior of such particles in the body, that a new discipline of 'nanotoxicology' was recently proposed [1].

Traditionally, particle size has played little part in evaluating health risk beyond establishing where in the respiratory tract inhaled particles will potentially deposit. Occupational aerosol exposure management is generally based on the assumption that health effects are primarily associated with the mass and bulk chemistry of inhaled material.

A number of studies carried out since the late 1980's have been showing a poor correlation between toxicity and mass alone when using relatively insoluble respirable particles $[2,3]$. Besinis et al. [4] were some of the first to demonstrate a particle size-dependence on toxicity, using titanium dioxide particles of different diameters.

Similar studies have shown that toxic response to inhaled micrometer-diameter low-solubility particles scales poorly with mass concentration, but closely with surface area concentration [5]. This research clearly implies that insoluble aerosols of very small particles having a high specific surface area are potentially more toxic than those comprised of larger particles. The term 'ultrafine aerosol' has been loosely adapted to differentiate aerosols dominated by sub- $100 \mathrm{~nm}$ diameter particles from those dominated by larger particles.

A number of nanotechnology-based materials are formed or used in the gas phase, and it is inevitable that the technology will lead to some workers being exposed to new and unique nano-sized and/or nanostructure particles. Singlewalled carbon nanotubes (SWCNTs) are a good example. Individual SWCNTs are relatively insoluble, on the order of 1.4 $\mathrm{nm}$ in diameter up to tens of micrometers long; with a specific surface area in excess of $3000 \mathrm{~m}^{2} / \mathrm{g}$ [6]. To summarize, significant progress has been achieved in the area of CNT processing. A range of new chemical treatments that are tailored in agreement with the desired application of the CNTs have demonstrated interesting results. Among them, covalently functionalized CNTs have been shown to improve both dispersion and polymer-CNT interaction. Avoiding damaging of the CNT structure upon chemical functionalization or purification procedure is still challenging [6]. Further, the exact influence of the pre-treatments on the chemical and physical properties of the CNTs is difficult to assess. Regarding CNT-based composites, the prevailing problems of dispersion and stress transfer are not completely being overcome. However, progress in this area has to be continued for the development of selective and innovative chemical treatments that will hopefully help for manufacturing of CNT-based devices and materials.

Gas-phase processes generally use transition metal catalyst particles on the order of a few nanometers in diameter during production. The resulting unprocessed material is a matrix of nanotubes, nanometer-diameter catalyst particles such as iron or nickel, and carbonaceous material similar to carbon black. Early studies have been inconclusive on the toxicity of the material, although it does appear to be biologically active [7]. However, the material is so unique in our experience that appropriate protocols for resting its toxicity are still being worked out. CNTs are just a single example of the new materials that may be encountered in the workplace as nanotechnology progresses.

Nanotechnology offers the promise of many benefits to society. However, it also presents many challenges as for how we work responsibly with nanomaterials and devices and protect the health of workers. Fortunately, the research initiated by concerns over exposure to insoluble aerosols has laid a good foundation for addressing critical issues. While we do not have all the answers yet, we can start by asking appropriate questions. Sufficient toxicity data are available to raise concerns over exposure to nanostructured materials where appropriate. Although little is known about the efficacy of engineering controls, personal protective equipment and respiratory protection for nanostructured materials, accepted and validated theory provides a good starting point for developing appropriate health-related production and handling strategies.

Uniquely, we have an opportunity to develop appropriate risk management systems in parallel with a new technology. If the opportunity is not taken, we run the risk of endangering the health of workers. However, through international collaborations and partnerships among researchers, industry, policymakers and other stakeholders aimed at understanding and controlling the risks associated with exposure to nanoscale materials, we have the potential to facilitate the development and implementation of what has been termed 'responsible nanotechnology'.

\section{Nanotoxicity}

Riehemann [8] had investigated the effect of the CNTs on the conformation and function of proteins by using the large scale molecular dynamics simulations. WW and SH3 domains were used as examples to illustrate that the CNTs can affect the function of the proteins by either disrupting the structure of active site or shielding the active site from competing ligands. In the simulation of the SWCNT and the WW domain, it was found that the SWCNT can plug into the hydrophobic core of WW domain to form a stable protein-SWCNT complex. 
This insertion of the SWCNT broke the scaffold which used to bind the proline rich motifs (PRM), and thus reduced the possibility of the direct binding between the PRM and the WW domain. In the case of SH3 domain, Riehemann studied a three-way binding competition among the SWCNT, the PRM ligand, and the SH3 domain, and found that the SWCNT had a very high probability occupying the binding pocket of the ligand in the $\mathrm{SH} 3$ domain, with about $0.6 \mathrm{kcal} / \mathrm{mol}$ more favorable binding affinity than the original PRM ligand. Moreover, in most of the simulation trajectories, the ligand will be adsorbed onto the SWCNT, which may further reduce the possibility of the correct binding for the ligand onto the active site of the SH3 domain. These adverse effects of the SWCNTs on proteins, including both the disruption of the active sites and the competitive binding with the ligands, might seriously damage the original functions of proteins, suggesting the potential nanotoxicity of the SWCNT. The interactions between the SWCNTs and the hydrophobic residues, particularly the aromatic residues ( $\pi-\pi$ stacking interactions), are found to play a key role in both the insertion of the SWCNT and the competitive binding with ligands. Moreover, Riehemann also simulated much larger systems, with human serum proteins interacting with SWCNTs, and found a competitive binding among all these serum proteins, with the adsorption capacity in an order of BFg $>\gamma-\mathrm{lg}>\mathrm{Tf}>$ $B S A$, in good agreement with experimental findings. Again, the $\pi-\pi$ stacking interactions are found to be the driving forces for the competitive binding of human serum proteins onto SWCNTs. These findings might have shed some light to the general mechanism of the interactions between hydrophobic nanoparticles and proteins.

\section{Outcomes and Suggestions}

The development in CNTs manufacturing using preparative methods, characterization data, and possible applications of conducting polymer (CPs)/CNTs composites will open the door for further application in the space filed and toxicity science. The electrical, thermal, mechanical and electrochemical properties of the composites in general are categorized intermediately between pure polymer and CNTs but vary depending on the method of preparation, type, purity, content and the dispersion of CNTs in the polymer matrix. In particular, the composite reveals synergistic effects and new properties which account for the interaction between CPs and CNTs at nanoscale. The effect offers an attractive route to create new multifunctional materials with great potential in uses involving mechanical, thermal, electrical, electrochemical features. However, the nature of the CP/CNT interaction and its effect on overall properties of the system still are unclear and need to further exploit and develop into practical application.

\section{References}

1. Bello D, Warheit DB. Biokinetics of engineered nano-TiO2 in rats administered by different exposure routes: implications for human health. Nanotoxicology.2017;11(4):431-433.doi:10.1080/17435390.2017.1330436

2. Lundgren DL, Hahn FF, McClellan RO. Toxicity of $90 \mathrm{Y}$ in relatively insoluble fused aluminosilicate particles when inhaled by mice. Radiat. Res. 1981; 88(3): 510-523. doi: $10.2307 / 3575640$

3. Poland C. Understanding the basis of pulmonary toxicity of high aspect respirable particles. Toxicol. Lett. 2014; 229: S11. doi: 10.1016/j. toxlet.2014.06.069

4. Besinis A, Hadi SD, Le HR, Tredwin C, Handy RD. Antibacterial activity and biofilm inhibition by surface modified titanium alloy medical implants following application of silver, titanium dioxide and hydroxyapatite nanocoatings. Nanotoxicology.2017;11(3):327-338.doi:10.1080/17435390.2017.1299890

5. Oosterwijk MTT, Feber LF, Burello E. Proposal for a risk banding framework for inhaled low aspect ratio nanoparticles based on physicochemical properties. Nanotoxicology. 2016; 10(6): 780-793. doi: 10.3109/17435390.2015.1132344

6. Aljerf L, Nadra R. Developed greener method based on MW implementation in manufacturing CNFs. Int. J. Nanomanufacturing. 2018; 14.

7. Liu J, Sonshine DA, Shervani S, Hurt RH. Controlled release of biologically active silver from nanosilver surfaces. ACS Nano. 2010; 4(11): 6903-6913. doi: $10.1021 / \mathrm{nn} 102272 \mathrm{n}$

8. Riehemann K. Nanotoxicity: How the body develops a way to reduce the toxicity of carbon nanotubes. Smll. 2012; 8(13): 1970-1972. doi: 10.1002/ smll.201200400 